Article

\title{
Hall-Effect Current Sensors Susceptibility to EMI: Experimental Study
}

\author{
Orazio Aiello (D) \\ Department of Electronics and Telecommunications (DET), Politecnico di Torino, Corso Duca degli Abruzzi 24, \\ I-10129 Torino, Italy; orazio.aiello@polito.it
}

Received: 27 September 2019; Accepted: 3 November 2019; Published: 8 November 2019

check for updates

\begin{abstract}
The paper deals with the susceptibility to Electromagnetic Interference (EMI) of Hall-effect current sensors. They are usually employed in power systems because of their galvanic isolation. The EMI robustness of such contactless device was compared with that of resistive current sensing (wired method). To this purpose, a printed circuit board (PCB) was fabricated. EMI tests methods such as Bulk Current Injection (BCI), Transverse-Electromagnetic (TEM) cell and Direct Power injection (DPI) were performed to evaluate the robustness of the Hall-Effect current sensor. EMI-induced failures are highlighted by comparing the different measurements tests and setups.
\end{abstract}

Keywords: hall-effect current sensors; commercial current sensor; electromagnetic compatibility (EMC); electromagnetic interference (EMI); direct power injection (DPI) test; transverse-electromagnetic (TEM) test; bulk current injection (BCI) test

\section{Introduction}

Current sensing circuits are essential for control and monitoring purposes in switching power supplies. In fact, a current sensor is usually employed in the current-mode loop control of DC-DC converters [1-5]. Among the current sensing techniques, Hall-effect current sensors are sensitive to the magnetic field generated by the current to be detected. This allows keeping the power circuit current flows to be monitored electrically isolated from the sensor [6]. On this basis, Hall-effect sensors are not affected by conducted interference generated by the power system to be monitored [7-10] and are therefore particularly employed in electromagnetically-polluted environments.

In this study, the correctness of Hall-effect current sensor operation in the presence of radiated and conducted Electromagnetic Interference (EMI) was investigated referring to a commercial device. A printed circuit board (PCB) suitable to perform Electromagnetic Compatibility (EMC) test was designed so that a continuous wave (CW) Radio Frequency Interference (RFI) could be superimposed on an operating Hall-effect current sensor according to the respective international standard (such as Bulk Current Injection (BCI) [11], Transverse-Electromagnetic (TEM) cell [12] and Direct Power injection (DPI) [13]).

The paper is organized as follows. In Section 2, the test printed circuit board (PCB) is described. Section 3 focuses on the $\mathrm{BCI}$ experimental setup. The results of the respective $\mathrm{BCI}$ immunity tests are also reported and discussed. In Section 4, the EMI susceptibility of the Hall-effect is further investigated by TEM cell immunity tests. The DPI tests performed to point out the Hall-effect susceptibility to conducted disturbances are presented in Section 5. Finally, some concluding remarks are drawn in Section 6.

\section{Test Board}

To compare the EMI robustness of a contactless current sensing approach (that exploits the Hall-effect) and a wired one (based on the voltage drop across a sensing resistance), a test PCB was 
designed. Whenever the Power MOS is switched-on by a driver, the current to be detected flows through the power trace on the PCB. When the setup aims to test a resistive current sensing under the effect of EMI, the Hall sensor output is floating and the voltage across the sense resistor $R_{\text {sense }}$ in series with the Power MOS is properly amplified and processed by a detection block (as sketched in Figure 1a). On the contrary, to evaluate the EMI robustness of the Hall sensor, the resistor $R_{\text {sense }}$ is shorted and the output voltage of the Hall sensor is elaborated by a properly-modified detection block (as sketched in Figure 1b).
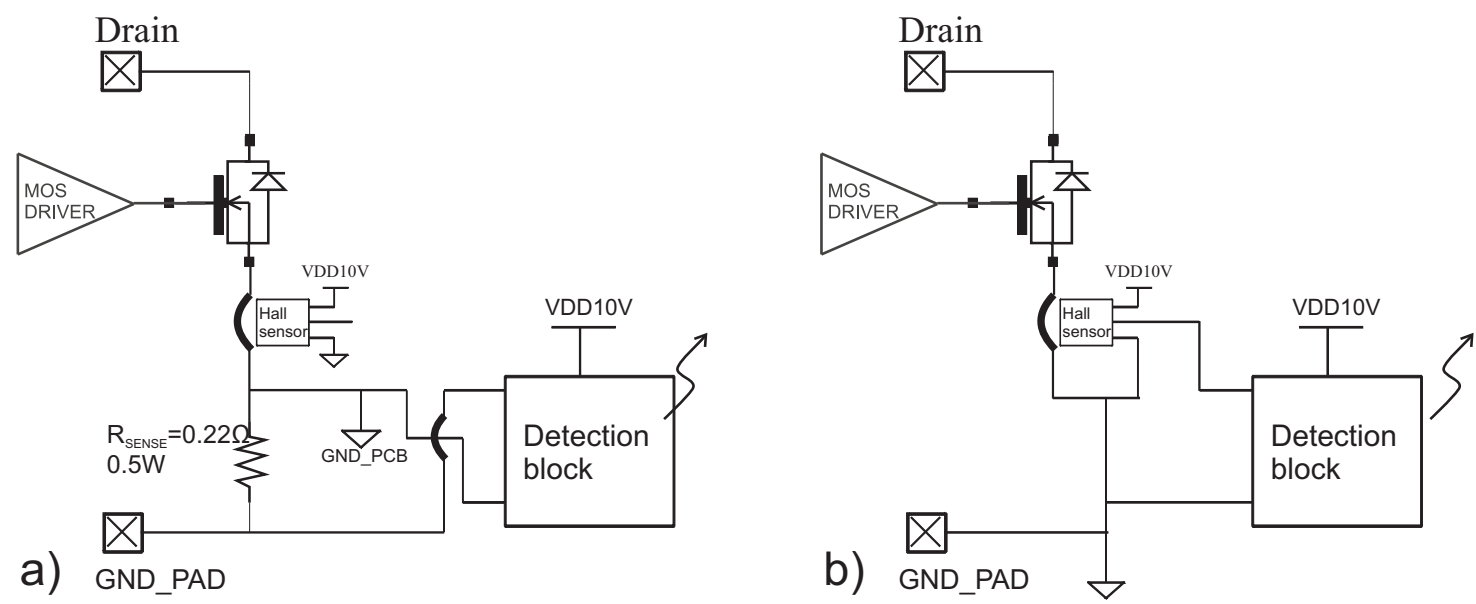

Figure 1. Schematic view of the current sensing setup for: (a) resistive approach; and (b) the Hall sensor.

A schematic layout representation of the the test PCB that can address both the current sensing methods is reported in Figure 2. A power MOS transistor, which is remotely driven by an optical fiber link, is employed to switch on and off the power circuit. The power supplies to the MOS and to the analog front-end are obtained from a line of the wiring harness and reach the PCB trough an automotive connector.

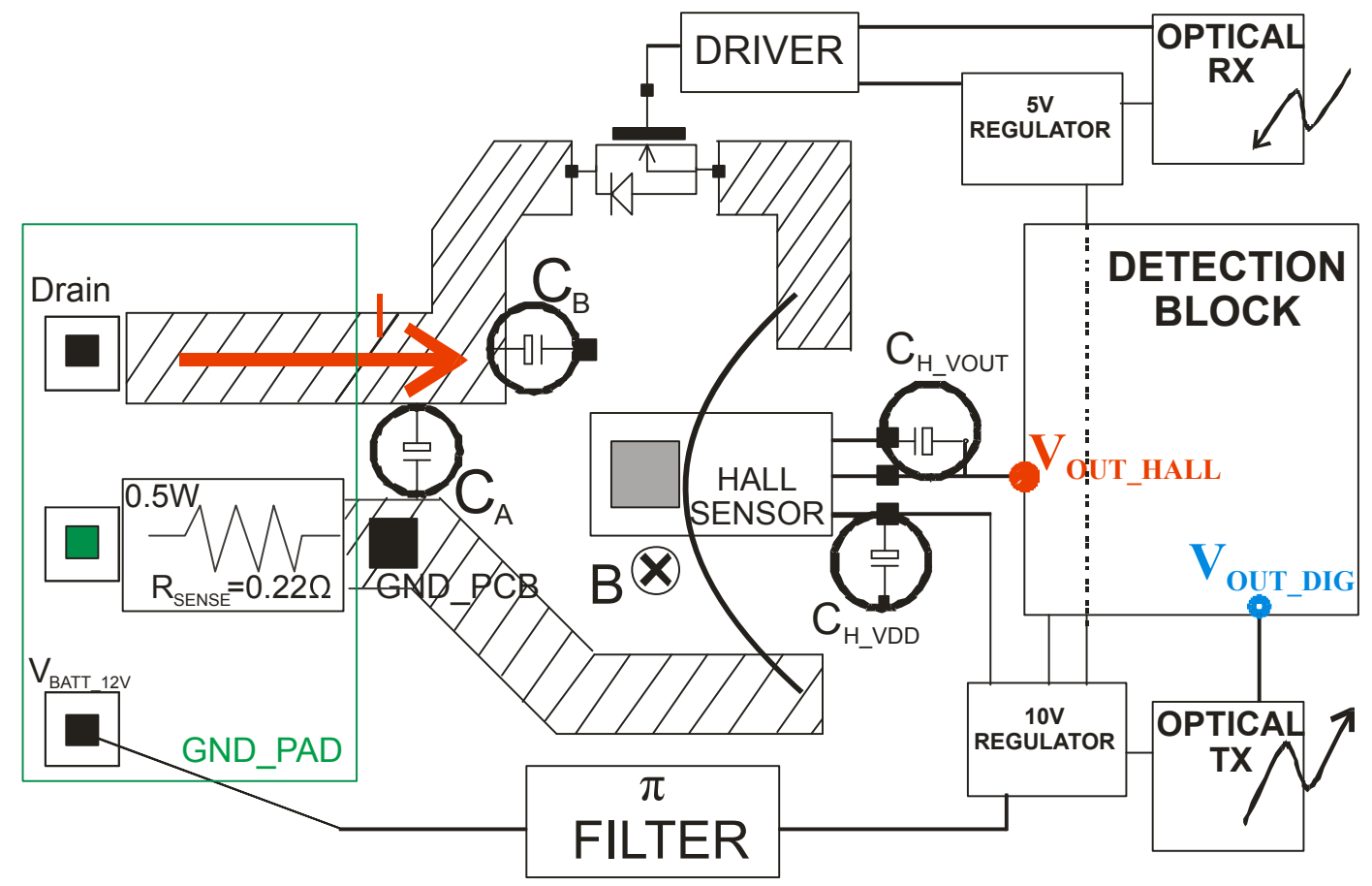

Figure 2. Layout representation of the testing board. 
The power supply voltage for the signal acquisition front-end is filtered by a $\pi$-type LC and further processed by a $10 \mathrm{~V}$ regulator. To reject conducted disturbance in the range of the DPI tests (1-400 MHz), the $\pi$ filter is composed of a $3 \mu \mathrm{H}$ inductor and two $100 \mathrm{nF}$ capacitors at its terminals. The regulator provides the power supply to the Hall sensor, to the optical transmitter and to the detection block. A further voltage regulator provides a $5 \mathrm{~V}$ supply to the optical receiver and power driver.

The bottom side of the PCB is a ground reference plane named GND_PCB. In Figure 2, the rectangular box on the left indicates a track on the bottom side of the PCB that separates two different ground plane references: GND_PAD and GND_PCB (respectively, inside and outside the rectangular box). This two reference areas are electrically connected by the resistance $R_{\text {sense }}$ during resistive current sensing, while, for the Hall-effect based current sensing, the two areas are shorted. Electromagnetic simulations by means of ANSYS Maxwell [14] were performed on the PCB cross section to ensure that the Hall-effect device did not suffer from the magnetic field due to the current in the bottom ground plate. Thus, only the wire placed on the Hall sensor (as in Figure 2) generated the magnetic field due to the current flowing in itself and to be detected by the sensor.

\section{Hall-Effect versus Resistive Setup}

Depending on which of the two considered current sensing is investigated (Hall-effect based or resistive one), proper paths are enabled and the respective components are mounted on the PCB to define the signal processing chain. Thus, the Hall sensor output voltage or the voltage drop across $R_{\text {sense, }}$, respectively, is properly processed by a detection block, as represented in Figure 2, which contains a non-inverting gain stage and a hysteresis threshold comparator, as reported in Figure 3. Such a hysteresis comparator provides a digital output voltage related to its threshold input voltages (output voltage of the amplifier), respectively, equal to $V_{T L}$ and $V_{T H}$. Such a comparator drives an optical transmitter so that the comparator output can be remotely monitored.

For resistive current sensing, these hysteresis thresholds correspond to a sensed current, respectively, equal to $I_{\min }=0.85 \mathrm{~A}$ and $I_{\max }=0.65 \mathrm{~A}$, as reported in Figure $4 \mathrm{a}$.

For Hall-effect current sensing, depending on the magnitude of the current flowing through the power circuit, a magnetic induction field is generated. The Hall-effect sensor is sensitive to this magnetic induction field and generates a proportional output voltage showing. The sensitivity is $1 \frac{\mathrm{mV}}{\mathrm{mT}}$, which roughly corresponds to an output voltage of $10 \mathrm{mV}$ for a current of $1 \mathrm{~A}$ flowing in the PCB power circuit. On this basis, the two voltage levels $V_{T L}$ and $V_{T H}$ are due to two correspondent current values equal, respectively, to $I_{\min }=0.9 \mathrm{~A}$ and $I_{\max }=2.7 \mathrm{~A}$, as reported in Figure $4 \mathrm{~b}$. Notice that these two current values vary depending on the distance between the Hall magnetic sensitive plate and the wire in which flows the current that generates the sensed magnetic field.

The hysteresis windows in Figure $4 \mathrm{a}, \mathrm{b}$ are defined to find a failure event in the current sensing induced by the EMI-presence. Such event corresponds to the High-to-Low transition of the hysteresis comparator that, in turn, produces a light signal generated by the optical RX that can be remotely acquired.

Despite of the fact that the hysteresis window amplitude for resistive current sensing is $10 \times$ narrower than the one employed with the Hall sensor (as can be highlighted comparing Figure $4 a, b$ ), the resistive current method shows a strong robustness to EMI. No failure in its current detection were experienced during any of the EMI tests discussed in this paper. Thus, for the sake of simplicity, the robustness of the resistive current method is not mentioned again so that only the investigations referring to the Hall-effect current sensor are discussed in the following. 


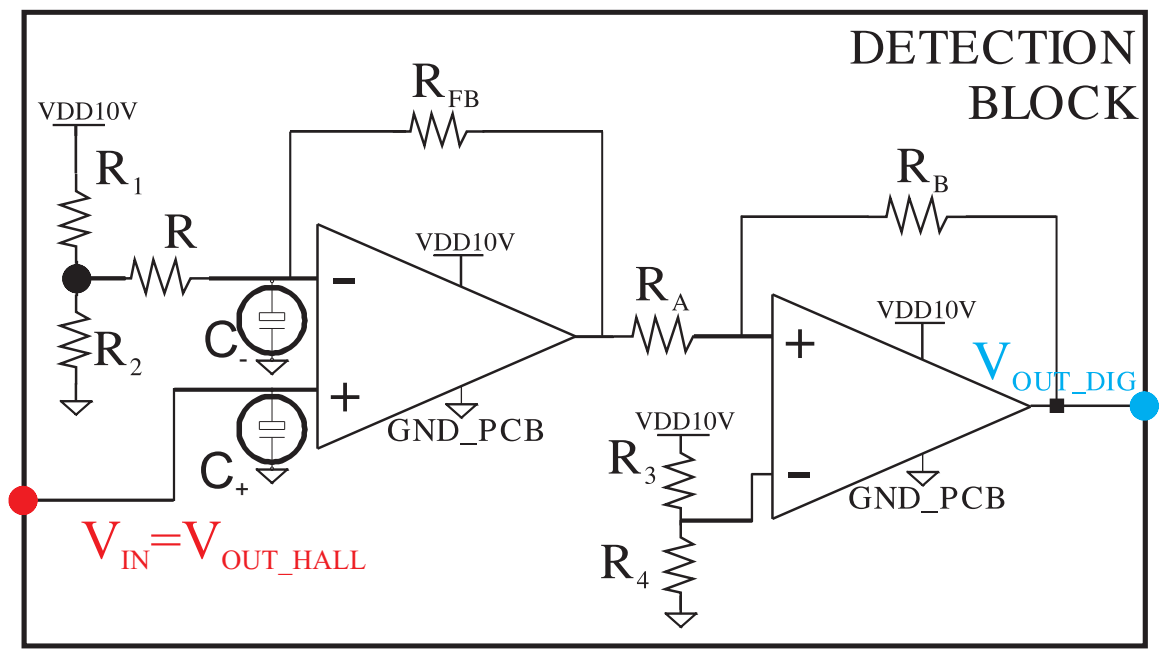

Figure 3. Processing chain of the Hall sensor output voltage.
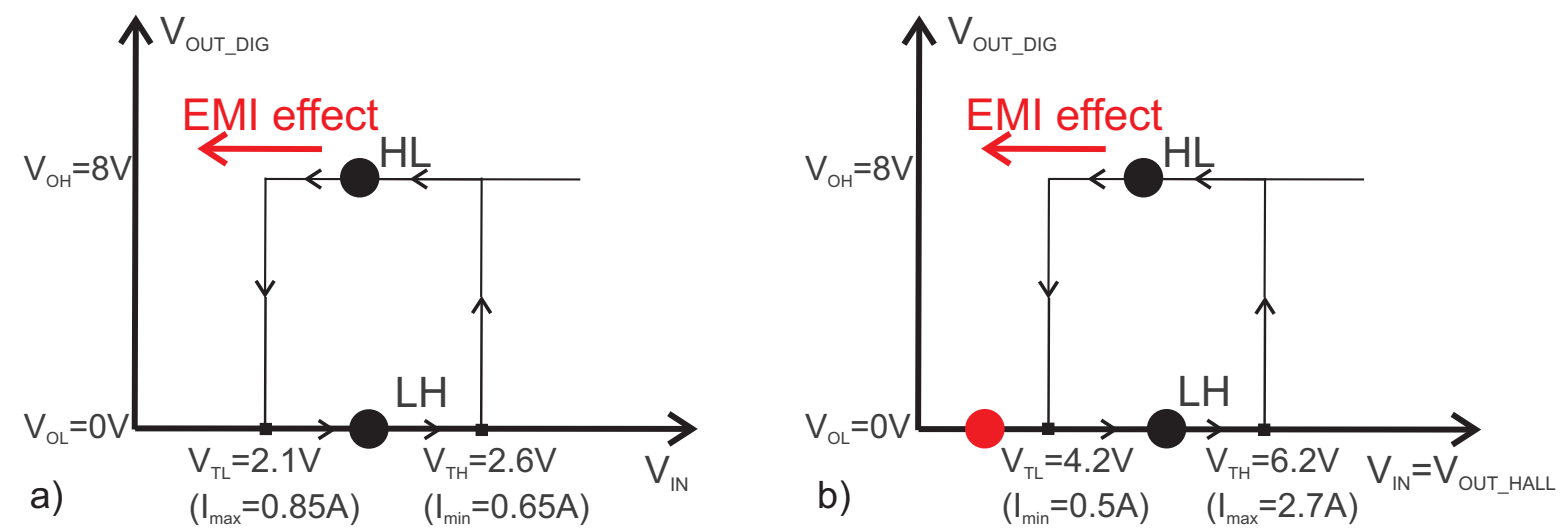

Figure 4. Hysteresis window of the output comparator for: (a) resistive current sensing; and (b) the Hall-effect sensor.

\section{Bulk Current Injection (BCI) Test}

To assess the susceptibility of the testing board introduced in Section 2, BCI tests were performed on the overall current detection system in Figure 5 by the setup in Figure 6 [11].

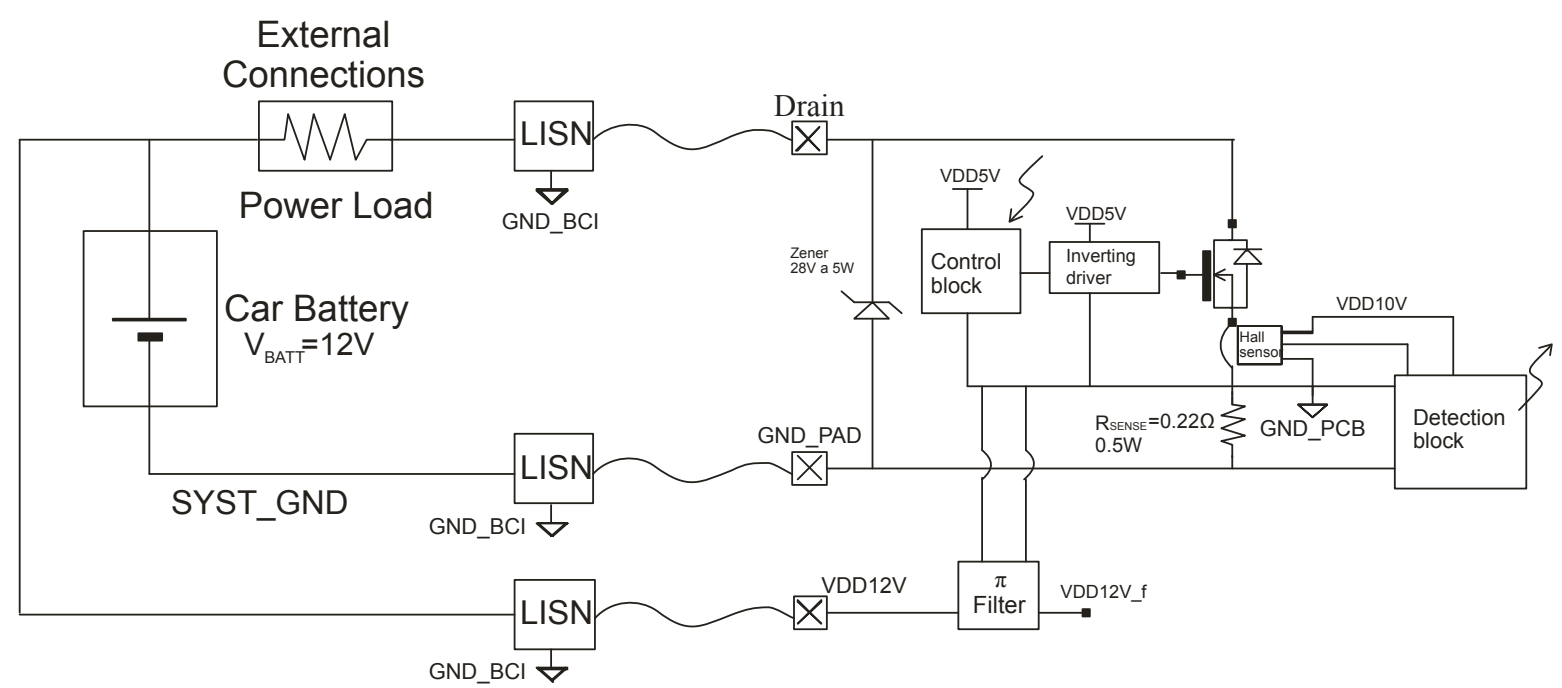

Figure 5. Overall schematic view of the current detection setup. 


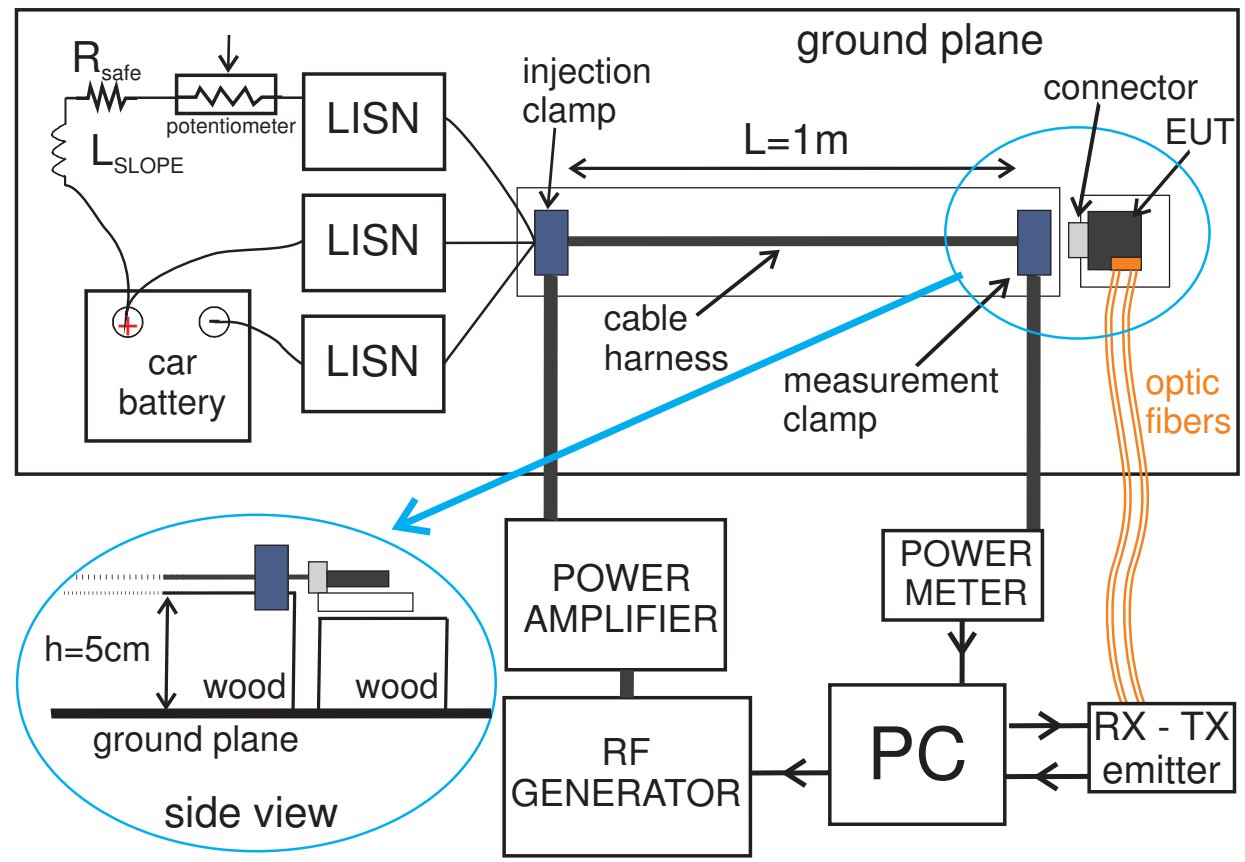

Figure 6. Pictorial representation of BCI test setup [11].

In such a setup, the equipment under test (EUT) is connected to the a variable resistive power load (potentiometer in the figure), to a car battery and to a remote ground reference through a $1 \mathrm{~m}$-long three-wire harness running $5 \mathrm{~cm}$ above the system ground plane on a wooden structure (inset in Figure 6). The three wires of the harness are connected to the loads and to the battery through three Line Impedance Stabilization Networks (LISNs in Figures 5 and 6), which provide a $50 \Omega$ RF termination to each line. Every LISN is designed to show an impedance equal to $50 \Omega$ at its $\mathrm{RF}$ terminals independently of the load connected at its low-frequency input terminals in the test bandwidth $(0-400 \mathrm{MHz})$.

During the tests, an injection clamp (F-130A-1 [15]) and a measurement clamp (F-51 [16]) by Fischer Custom Communication (FCC) are located along the harness, as shown in Figure 6, to perform $\mathrm{BCI}$ tests in compliance with [11]. The distance between the injection and measurement clamps is $75 \mathrm{~cm}$, while the distance between the measurement clamp and the EUT is $4.5 \mathrm{~cm}$. The injection clamp is connected to the output of an $10 \mathrm{~W}$ RF power amplifier, whose input terminal is connected to a CW RF signal source, while the measurement clamp is connected to an RF power meter to measure the bulk current injected into the EUT. The power amplifier, the RF source, the RF power meter and the control unit are located out of the test area. The EUT is connected to a two-way optical fiber link in order to drive the power transistor in the EUT and to monitor the current sensor output without perturbing the surrounding electromagnetic environment. A potential malfunction of the current detecting of the EUT during BCI tests is due to common-mode RF current (bulk current) injected into the EUT through its wiring harness.

\subsection{BCI Susceptibility Tests}

BCI measurements in the bandwidth (10-400 MHz) were performed by a PC-based acquisition system, as represented in Figure 6. For each test frequency, the RFI amplitude is increased until a failure in the DUT operation is experienced or the maximum incident RF power deliverable by the amplifier is reached. In the first case, the failure injected bulk current obtained from the power meter measurement is acquired. Otherwise, no failure value is reported (missing points and respective connecting lines in Figures 7-10).

Referring to the above-mentioned EUT, the commutation of the hysteresis comparator voltage has been considered as a failure criterion and the potentiometer has been configured so that a DC 
current $I=\frac{I_{\min }+I_{\max }}{2}$ flows in the power circuit. More precisely, at each failure event, the EUT has been configured so that the hysteresis comparator operates at the point HL in Figure 4 with no EMI excitation.

To highlight the susceptibility of the Hall-effect sensors, $\mathrm{BCI}$ immunity tests were performed the on different configurations of the EUT introduced in Section 2.

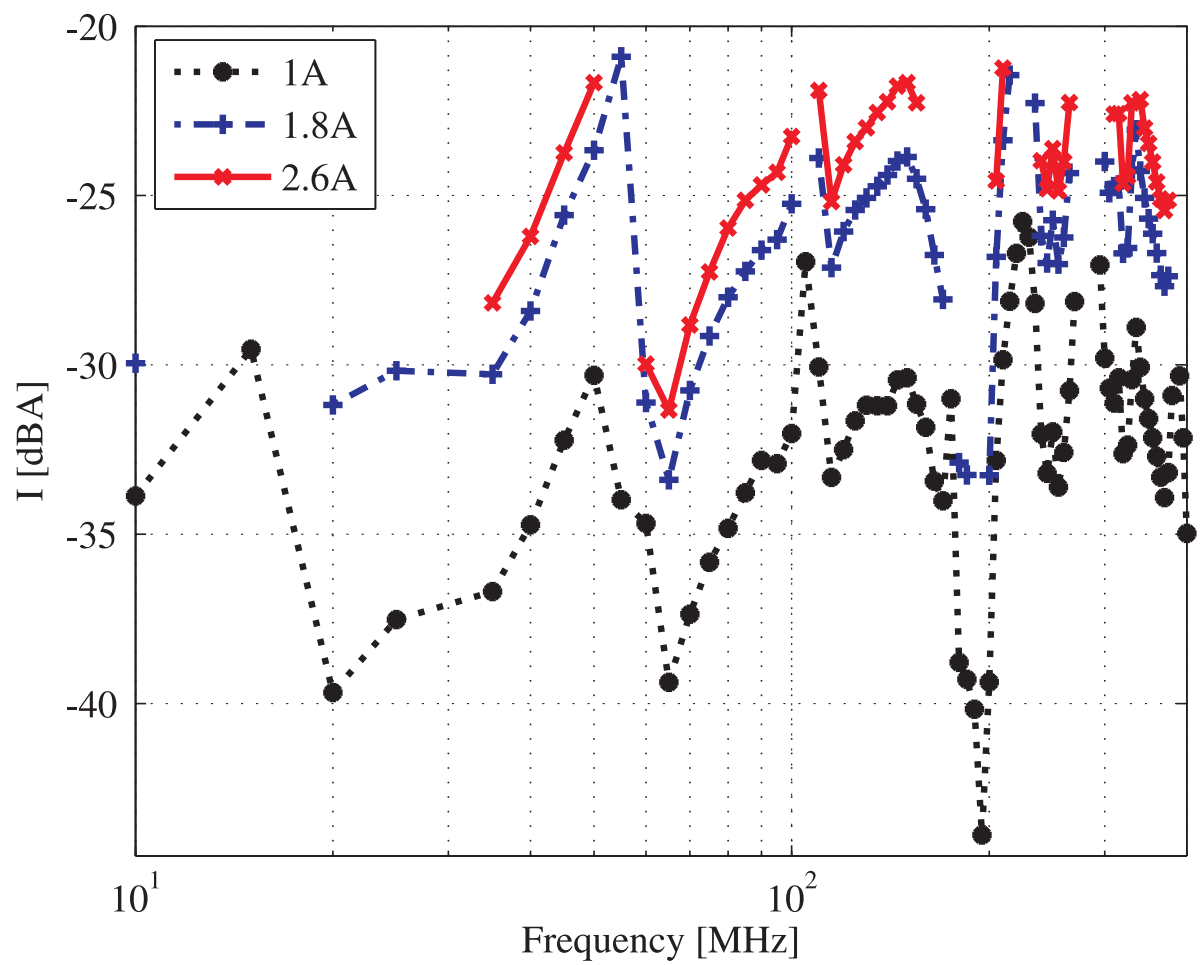

Figure 7. BCI immunity measurement for different current values.

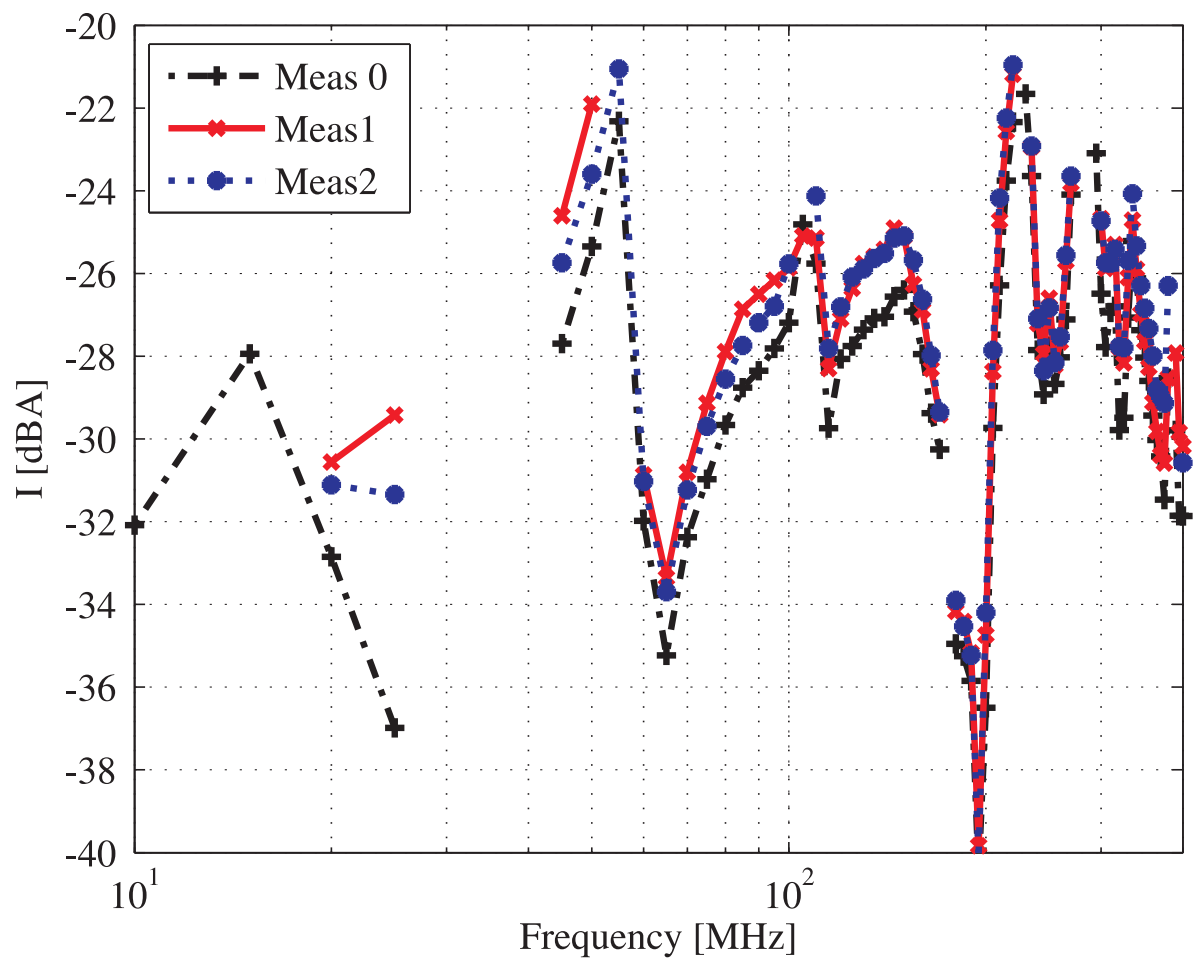

Figure 8. BCI immunity measurement for different caps presence in layout in Figure 2: Meas0 = no caps; Meas $1=C_{+}, C_{-}$; and Meas $2=C_{+}, C_{-}, C_{H_{-} V O U T}, C_{H_{-} V D D}$. 


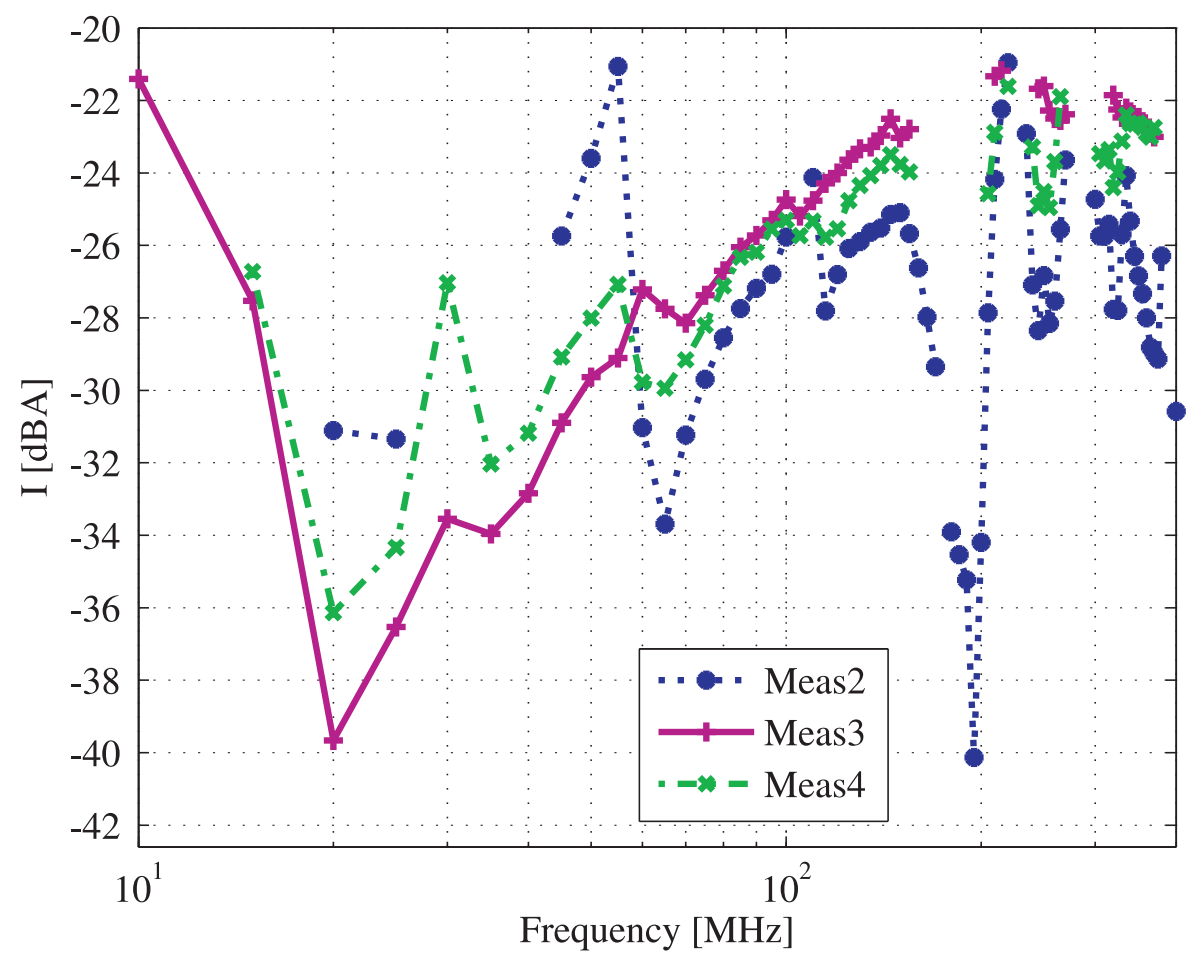

Figure 9. BCI immunity measurement for different caps presence in layout in Figure 2: Meas2 $=C_{+}$, $C_{-}, C_{H_{-} \text {VOUT }}, C_{H_{-} V D D} ;$ Meas3 $=C_{+}, C_{-}, C_{H_{-} V O u T}, C_{H_{-} V D D}, C_{A} ;$ and Meas4 $=C_{+}, C_{-}, C_{H_{-} V O u T}$, $C_{H_{-} V D D}, C_{B}$

\subsection{BCI Test Results}

The susceptibility to EMI of the EUT in Figure 6 was first tested for different values of the DC current flowing through the power line. The results of such tests are reported in Figure 7. It can be observed that the susceptibility level of the EUT scales with the DC current level, depending on the proximity of the threshold value.

Moreover, several BCI measurements were performed adding $100 \mathrm{nF}$ filter capacitors in order to reject the conducted disturbances. They were placed at the terminals of the main blocks (i.e., inputs of the detection block or Hall sensor leads) and to define the influence of the parasitic of the long trace on the PCB.

The encircle capacitances in Figures 2 and 3 point out the most significant filter capacitances added on the PCB in the successive tests described in this section. The susceptibility of the EUT with no filter capacitors on the Hall sensor acquisition front-end is firstly considered and named Meas0 in Figure 8. Then, two capacitors $C_{+}$and $C_{-}$were placed at the inputs of the operational amplifier of the detection block, as in Figure 3. The susceptibility tests were repeated and data collected as Meas1 in Figure 8. Another configuration, listed as Meas2 in Figure 8, includes the capacitors $C_{+}$and $C_{-}$as in Meas1 but two further capacitors $C_{H_{-} V O U T}, C_{H_{-} V D D}$ at Hall sensor leads, as represented in Figure 2. Comparing the above-mentioned three configurations, the capacitors do not strongly affect the immunity level of the EUT.

Other measurements were performed including other capacitors. The immunity level of the EUT was explored with a capacitor $C_{A}$ between the drain and source of the power transistor, as reported in Figure 2. At the capacitors already placed in Meas2, such capacitor $C_{A}$ was added and the respective measurements are named as Meas3 in Figure 9. Then, instead of the capacitor $C_{A}$, a capacitor $C_{B}$ between the drain and the ground plane on the bottom side of the DUT was placed. The respective measurements are reported as Meas4 in Figure 9. Comparing Meas2, Meas3 and Means4 shows that the respective additional capacitors result in a slightly higher immunity for frequencies higher than $600 \mathrm{MHz}$. Thus, no significant immunity improvement is experienced in any of the above-mentioned configurations. 
Other EMI investigations were performed for different Hall sensor inclination with respect to the PCB surface, as represented in Figure 10a. In all cases, the distance beetween the wire and the Hall sensor has not been changed. The experimental results obtained with such configurations are reported in Figure 10b and show a significant dependence of the susceptibility level on the inclination of the sensor.

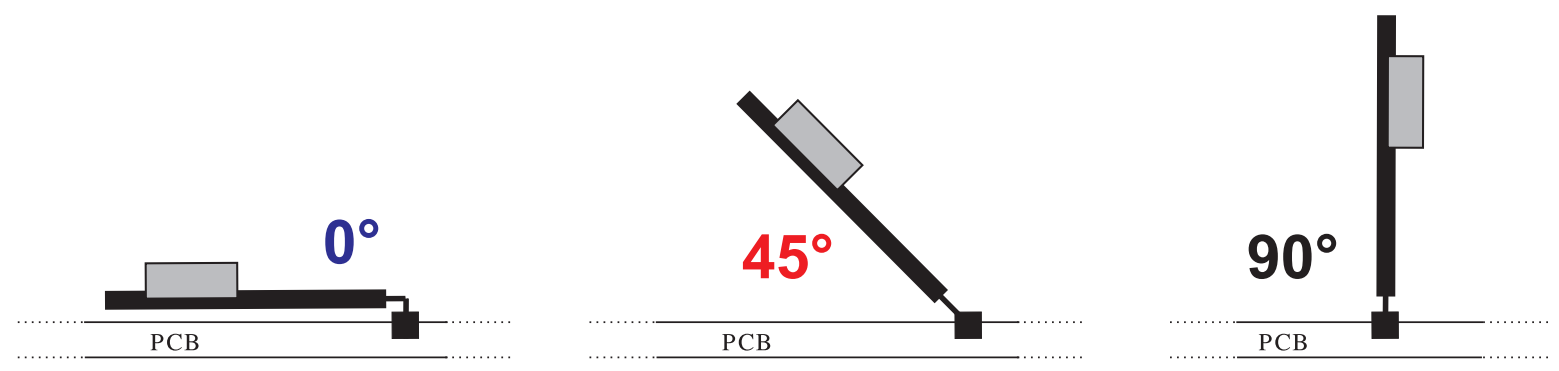

a)

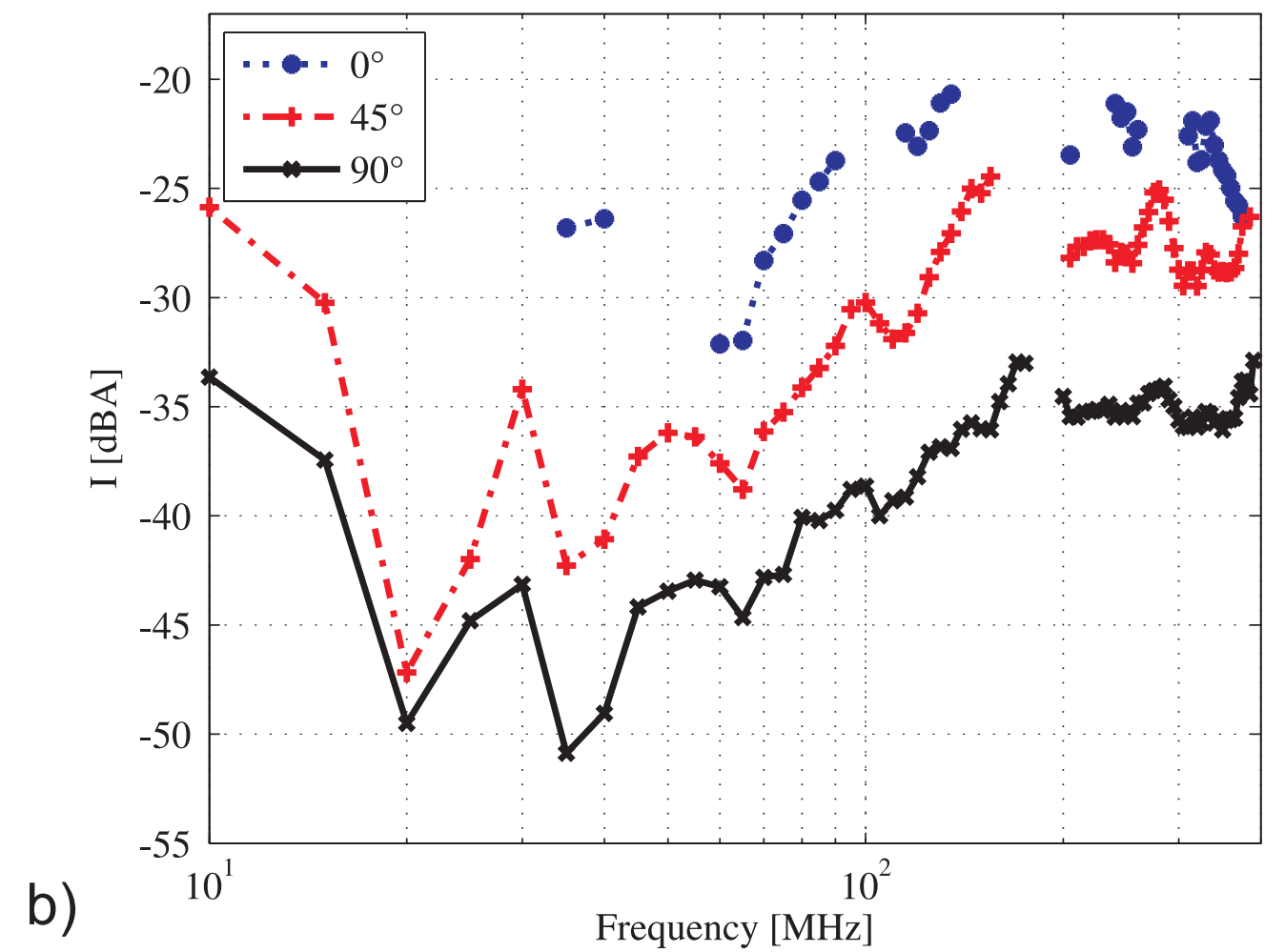

Figure 10. (a) Hall sensor inclination considered in BCI tests; and (b) respective BCI immunity measurements.

\subsection{Discussion}

Figure 7 shows how the failure level of the EUT scales with the current to be measured. As a consequence, EMI-induced failures seems to be related to an EMI-induced upset in the Hall-effect sensor output signal path. On the other hand, Figures 8 and 9 show that the susceptibility level of the EUT is scarcely affected by the introduction of filter capacitors at the conditioning amplifier inputs and at the terminals of the Hall-effect current sensors. Therefore, the EUT susceptibility is not related to the detection block or the trace parasitics. On the contrary, the measurements performed for different Hall sensor inclinations (Figure 10b) show that the immunity level is strongly affected by the inclination of the sensor. On this basis, the susceptibility to EMI of the EUT seems to be related to direct electromagnetic field coupling in the Hall-effect sensor body. For this reason, further TEM cell immunity tests were performed. 


\section{Transverse-Electromagnetic (TEM) Cell Tests}

To investigate in further detail the EMI-induced failures in the Hall sensor highlighted by BCI measurements, the susceptibility to radiated EMI of such a sensor was tested in a TEM cell, by placing the sensor in the cell, as sketched in Figure 11. The Hall sensor ground lead was shorted to the TEM cell internal side while the supply voltage (VDD) and the output $\left(V_{O U T}\right)$ terminals were AC-shorted to the internal side of the TEM cell. VDD and $V_{O U T}$ leads come out from the TEM cell upper side. Through these terminals, the DC power supply of the sensor was provided and its DC output voltage was measured. To verify the device susceptibility to electric and magnetic fields, the Hall sensor was placed inside the cell TEM in different orientations (A-D in Figure 11).

Figure 12 points out the magnetic and electric field directions in the different setup considered in the following. In Setups A and B, the RF electric field $E_{x}$ (along x-axis) is parallel to the device orientation. Instead, Setups $A$ and $B$ differ to the device orientation with respect to the RF magnetic field $H_{y}$ (along y-axis). In Setup A, $H_{y}$ is parallel to the Hall sensor surface, while, in Setup B, $H_{y}$ is orthogonal to the device. Similarly, in Setups $C$ and D, the RF electric field $E_{x}$ is orthogonal while these two setup differ to the RF magnetic field orientation with respect to Hall sensor surface.

\section{1. (TEM) Cell Measurement Result}

The radiated EMI susceptibility tests highlight that electromagnetic field distribution inside the TEM cell induces a negative offset in the Hall sensor output voltage. In Figure 13, the magnitude of the induced offset voltage in the range from $500 \mathrm{kHz}$ to $1 \mathrm{GHz}$ for an RF incident power applied to the TEM cell equal to $34 \mathrm{dBm}$. This implies a vertical electric field magnitude of $250 \mathrm{~V} / \mathrm{m}$ and a transversal magnetic field magnitude of $0.66 \mathrm{~A} / \mathrm{m}$ where the Hall sensor under test is placed [17-19].

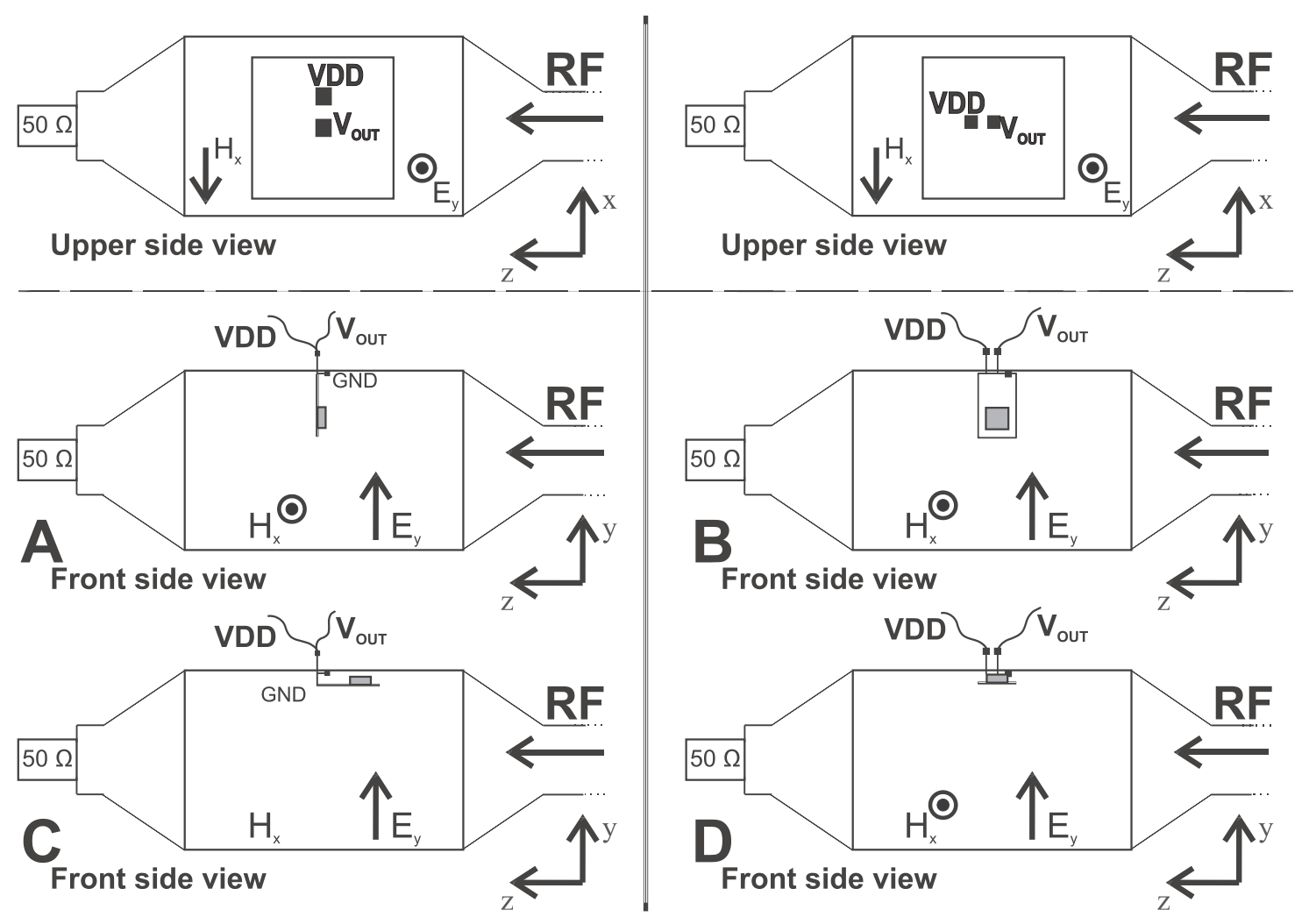

Figure 11. TEM cell testing setup. 


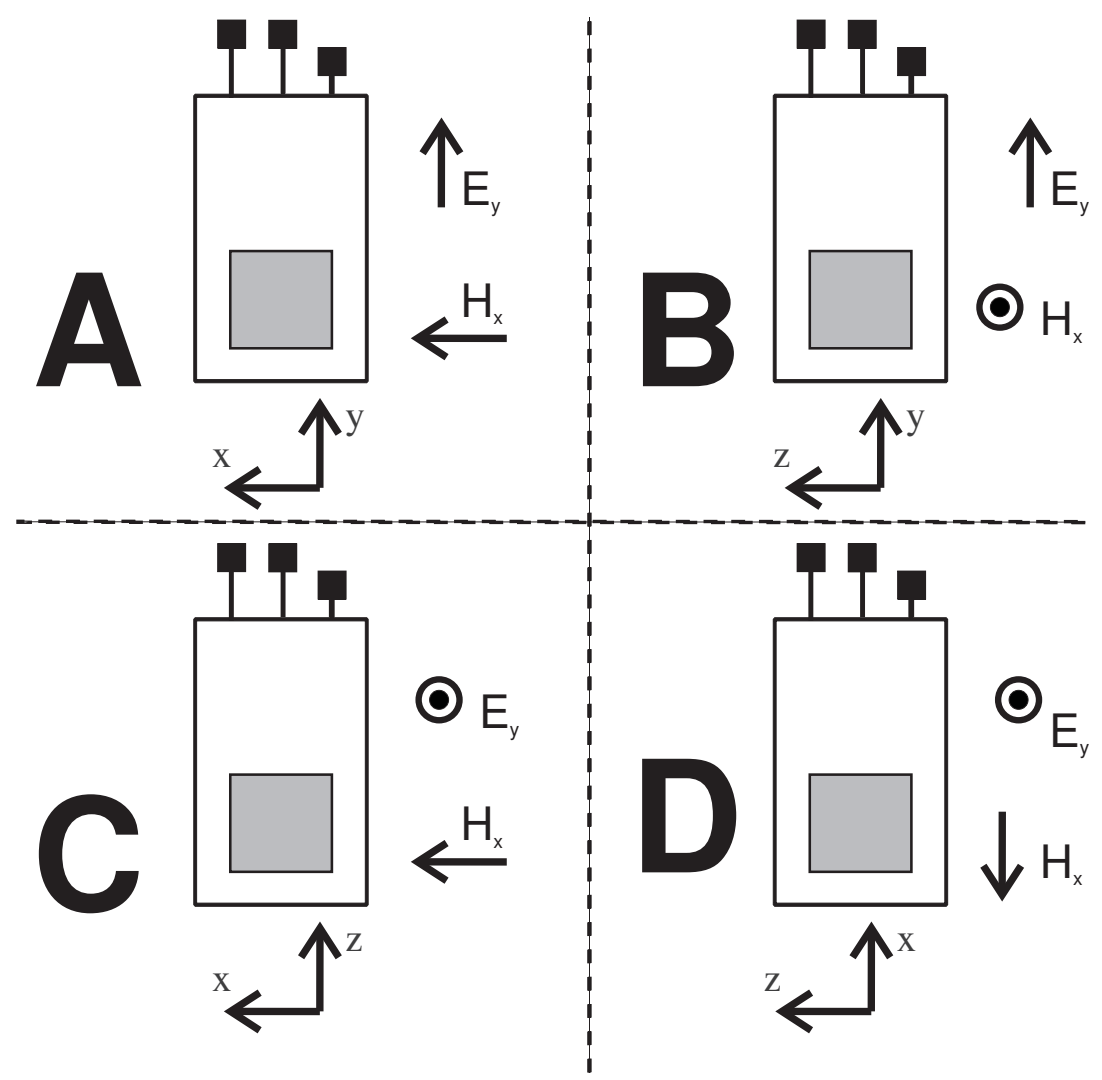

Figure 12. Magnetic and electric field orientation for different Hall sensor setup.

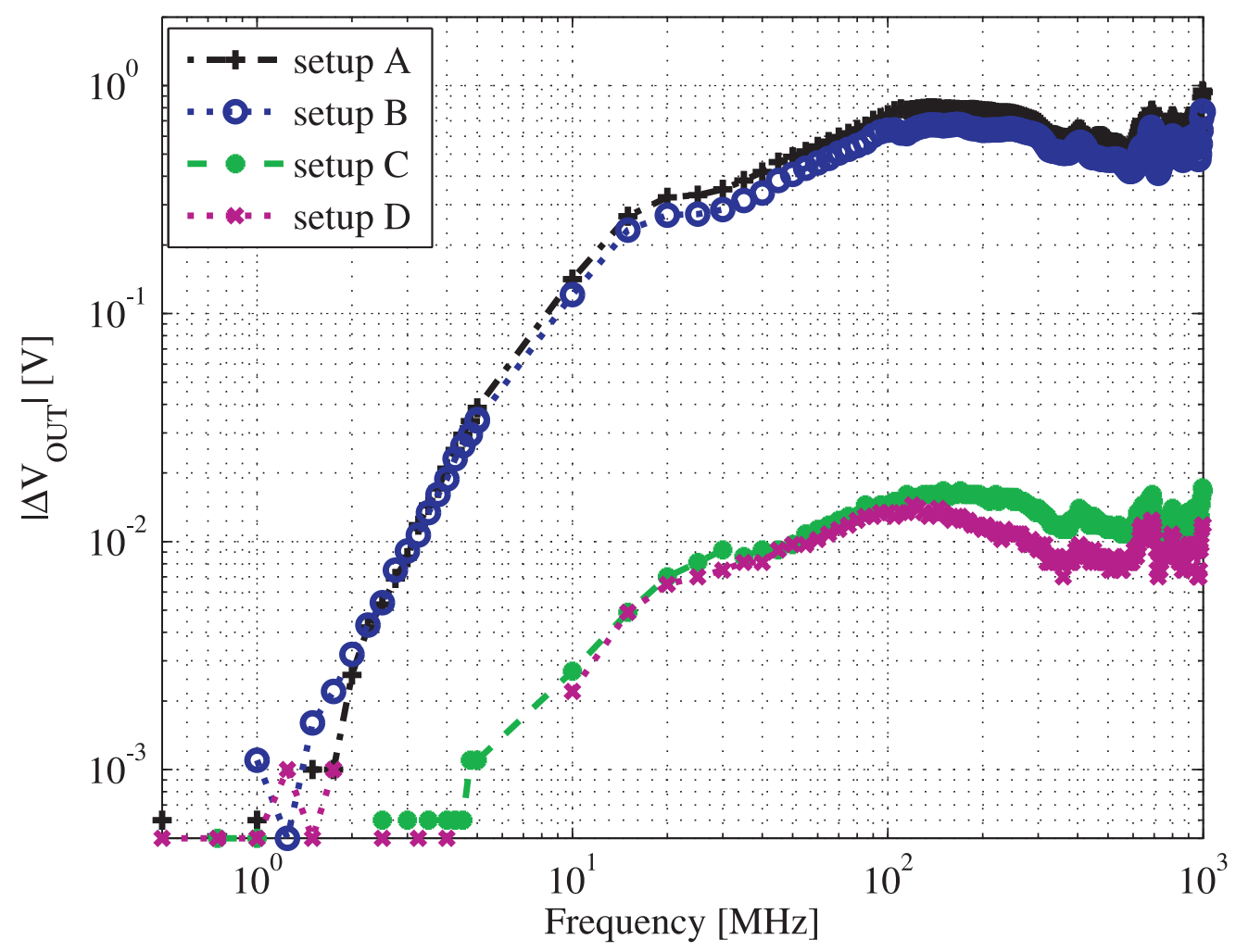

Figure 13. TEM cell immunity measurements at $34 \mathrm{dBm}$ RF power amplitude for different setup. 


\subsection{Discussion}

Figure 13 highlights the similarity between the EMI induced offset voltage measured in Setups A and B. In addition, Setups C and D show similarity in terms of EMI-induced offset. Moreover, Setups A and $\mathrm{B}$ are differently coupled to the TEM cell magnetic field and similarly coupled to the electric field, whereas Setups A and C (Setups B and D) are differently coupled in terms of electric field. On this basis, the susceptibility to EMI of the Hall-effect sensor can be related to the electric field parallel to the Hall sensor plate. This could be due to the susceptibility of the sensor either to the electric field directly coupled to the sensing element or to the disturbances connected by leads and metal interconnections on the sensor body.

\section{Direct Power Injection (DPI)}

As a further EMI investigation, direct power injection tests [13] on the employed Hall sensor were performed. RF power was injected on the supply voltage of the Hall sensor by means of an RF generator, an RF amplifier and a bias tee, as represented in Figure 14. The employed bias tee has a lower bandwidth limit equal to $30 \mathrm{MHz}$. Therefore, in the following figure, the measurement results are not reliable for a frequency lower that $30 \mathrm{MHz}$. At the end of the power amplifier a $-3 \mathrm{~dB}$ attenuator was placed for the safety of the RF amplifier. In fact, if the RF amplifier were accidentally not totally connected (open circuit condition) during the measurement operation, a reflection coefficient $|\Gamma|=1$ could damage the RF amplifier.

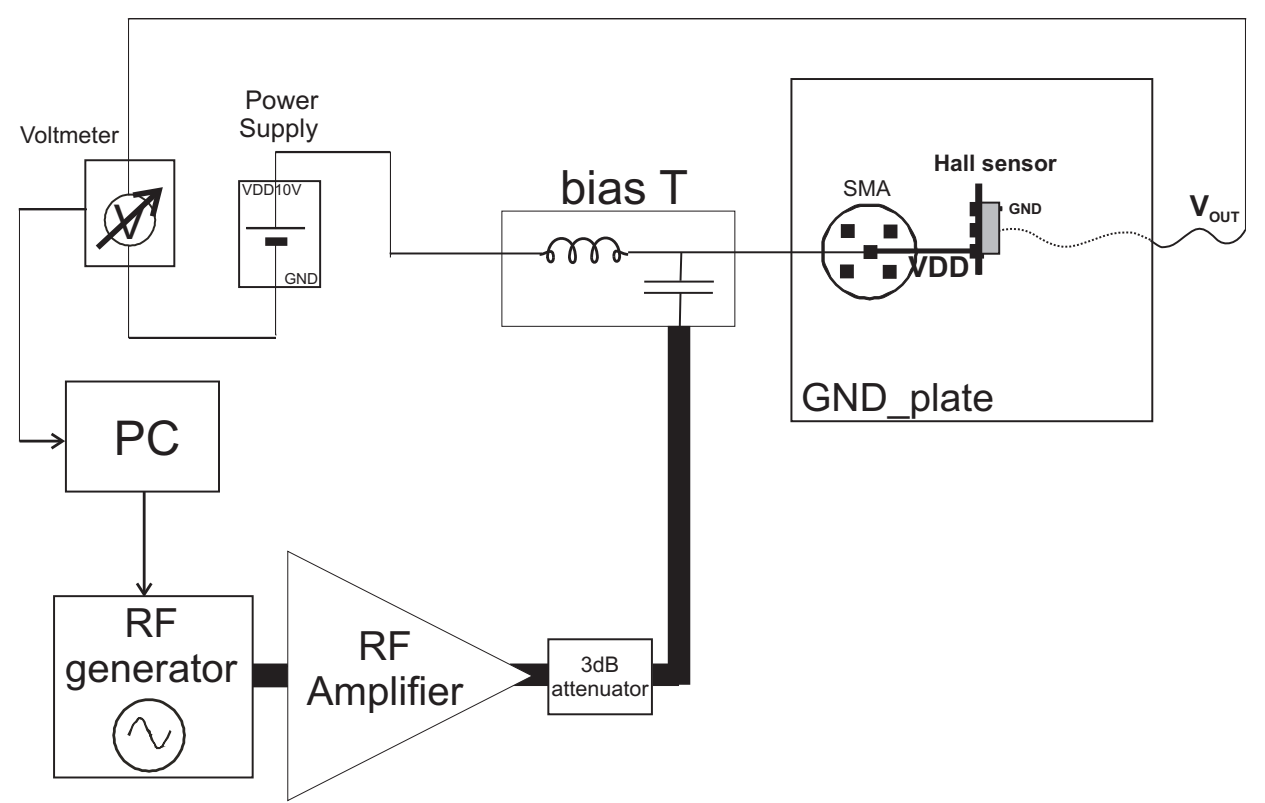

Figure 14. DPI on Hall sensor VDD Test setup [13].

The incident powers provided are given as results of the sum of the incident power of the RF generator (respectively, $-50,-40,-30,-20$, and $-10 \mathrm{dBm}), 34 \mathrm{dBm}$ of the RF amplifier and the attenuation due to a $-3 \mathrm{~dB}$ attenuator. On this basis, the induced offset voltages $\Delta V_{\text {OUT }}$ due to RF power amplitudes equal $-19,-9,1,11$, and $21 \mathrm{dBm}$ injected on VDD in the range $500 \mathrm{kHz}-1 \mathrm{GHz}$ are reported in Figure 15. Similarly, the induced offset voltages $\Delta V_{\text {OUT }}$ due to frequencies of $100 \mathrm{MHz}$, $500 \mathrm{MHz}$ and $1 \mathrm{GHz}$ for amplitude in the range $-19 \mathrm{dBm}$ to $+21 \mathrm{dBm}$ are reported in Figure 16 . An upper limit to provided RF power amplitude equal to $21 \mathrm{dBm}$ was chosen to avoid Hall sensor destruction due an excessive power at its terminals. Such an amplitude corresponds to a theoretical maximum voltage on supply voltage VDD lead equal to $17 \mathrm{~V}$. 


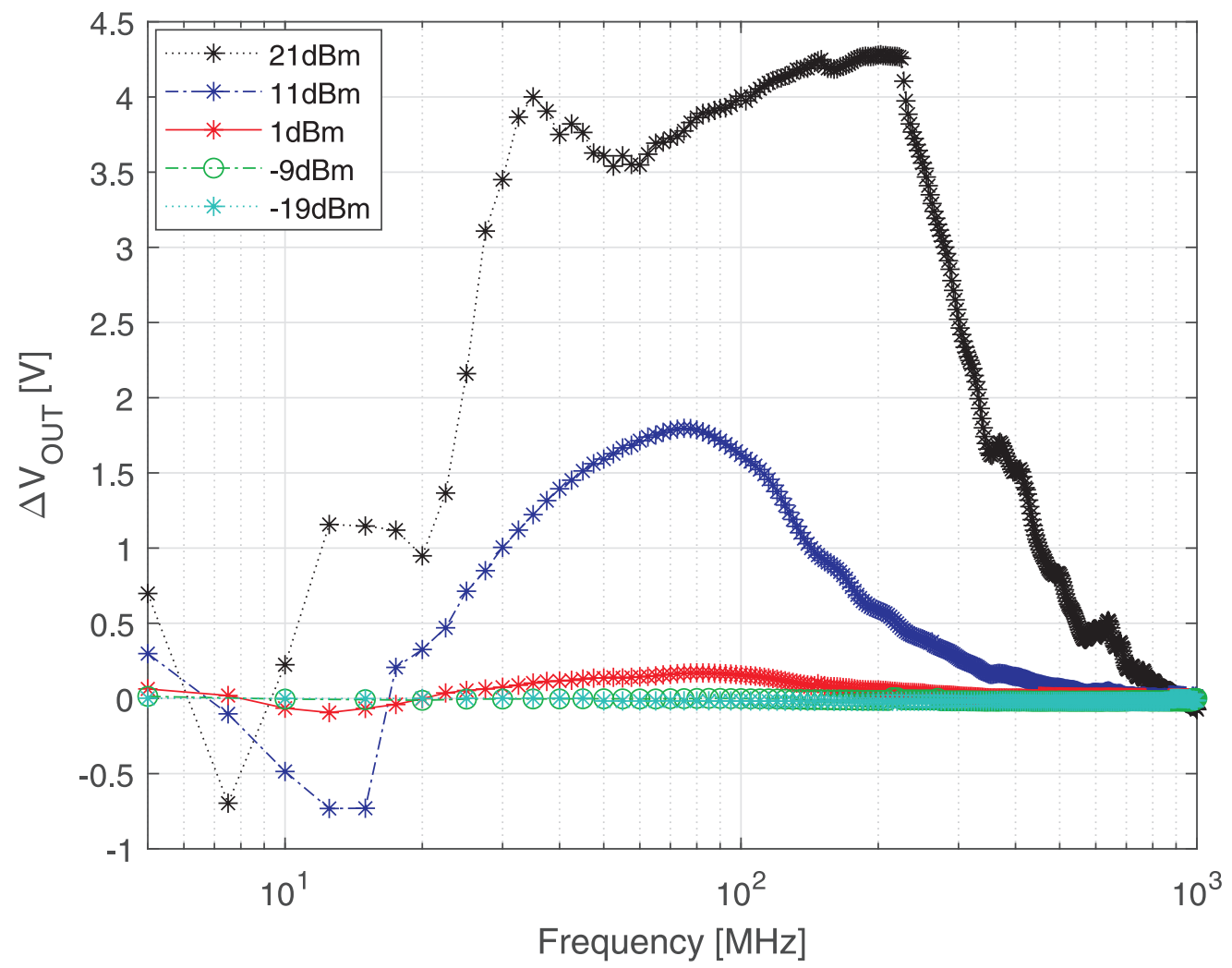

Figure 15. DPI on VDD immunity measurements ( $\left|\Delta V_{O U T}\right|$ vs. frequency) for different RF power amplitudes.

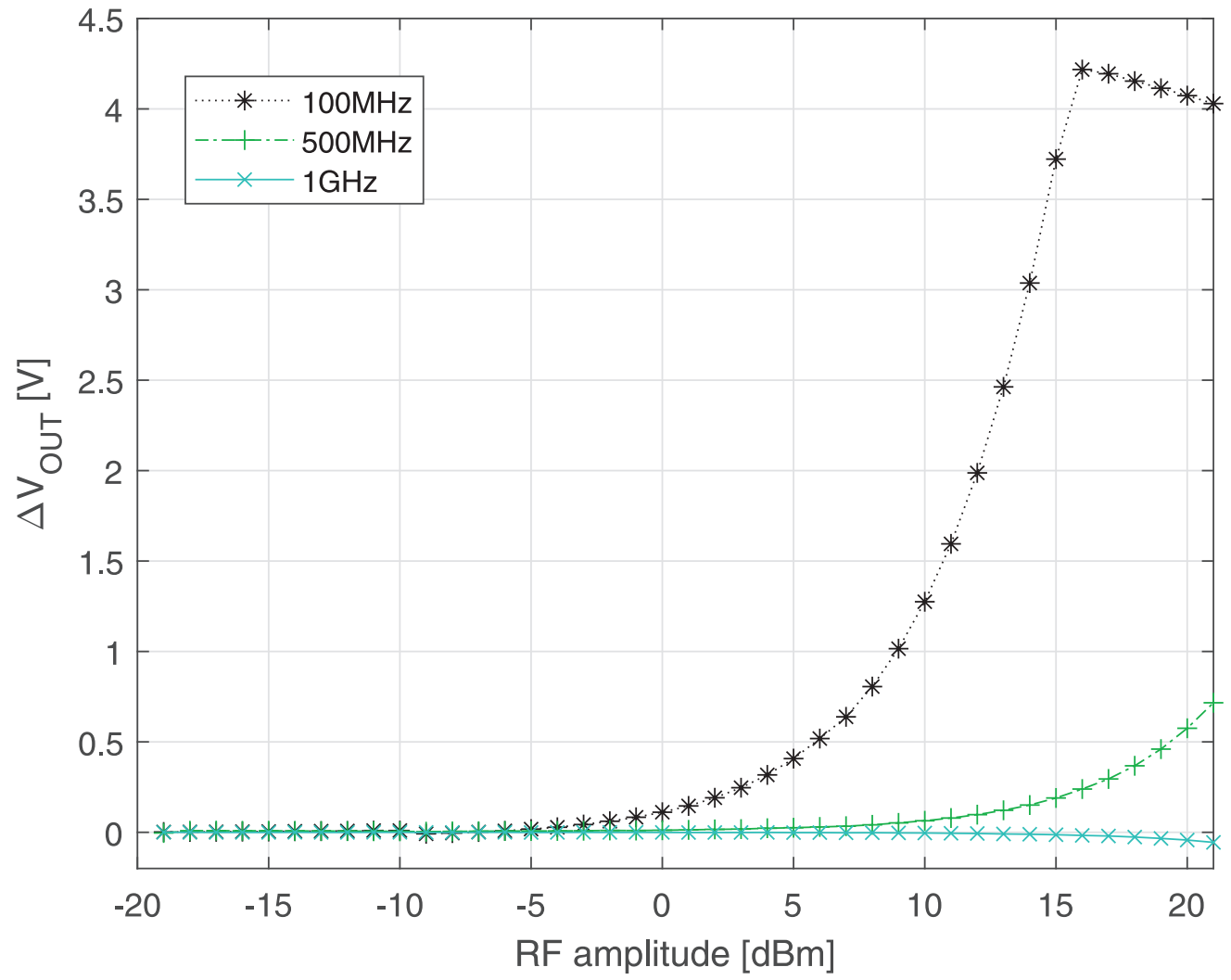

Figure 16. DPI on VDD immunity measurements ( $\left|\Delta V_{O U T}\right|$ vs. RF power amplitude) for different frequencies. 
Different signs of the induced offset are highlighted in Figures 15 and 16. These phenomena seem to be due to different nonlinear mechanisms of active elements in the device. Similar measurement results are shown even in the EMI superimposed on the output terminal of the Hall sensor. Although even the DPI tests show the susceptibility to EMI of the investigated Hall sensor, no precise reasons for such failure can be inferred.

\section{Conclusions}

The susceptibility to EMI of a Hall-effect sensor employed in current monitoring was investigated firstly referring to BCI test. Hall-effect current sensors (contactless) are dramatically affected by the EMI presence, contrary to the resistive (wired) current sensing method. BCI tests were performed in difference configurations, in addition to TEM cell and DPI tests. The measurements results show that a Hall-effect sensor can be strongly affected by the presence of EMI. In particular, the TEM cell tests highlighted how the operations of the specific Hall-effect sensor considered in this study are affected by an RF electric field excitation parallel to the surface of the sensing element. As the physical mechanism of the Hall-effect is not affected by a CW RFI [7], the failures of the Hall sensor are likely related to the ICs that interface the Hall sensor and process its signal. Although many investigations are present in the literature regarding amplifier and monitoring ICs [20-22], a precise understanding of the Hall sensor failure causes always relies on a complete knowledge of the ICs that interface with the Hall plates. This implies that the EMI-induced effects have to be taken into account from the first phases of the Hall-effect sensor design.

Funding: This research received no external funding.

Conflicts of Interest: The author declares no conflict of interest.

\section{References}

1. Tong, Q.; Chen, C.; Zhang, Q.; Zou, X. A Sensorless Predictive Current Controlled Boost Converter by Using an EKF with Load Variation Effect Elimination Function. Sensors 2015, 15, 9986-10003. [CrossRef] [PubMed]

2. Min, R.; Chen, C.; Zhang, X.; Zou, X.; Tong, Q.; Zhang, Q. An Optimal Current Observer for Predictive Current Controlled Buck DC-DC Converters. Sensors 2014, 14, 8851-8868. [CrossRef] [PubMed]

3. Aiello, O.; Fiori, F. A new mirroring circuit for power MOS current sensing highly immune to EMI. Sensor 2013, 13, 1856-1871. [CrossRef] [PubMed]

4. Aiello, O.; Fiori, F. Current sensing circuit for DC-DC converters based on the miller effect. In Proceedings of the 2013 International Conference on Applied Electronics (AE), Pilsen, Czech Republic, 10-11 September 2013; pp. 1-4.

5. Huang, K.; Liu, Z.; Zhu, F.; Zheng, Z.; Cheng, Y. Evaluation Scheme for EMI of Train Body Voltage Fluctuation on the BCU Speed Sensor Measurement. IEEE Trans. Instrum. Meas. 2017, 66, 1046-1057. [CrossRef]

6. Popovic, R.S. Hall Effect Devices, 2nd ed.; Institute of Physics Publishing: Bristol, UK; Philadelphia, PA, USA, 2004.

7. Aiello, O.; Fiori, F. A New MagFET-Based Integrated Current Sensor Highly Immune to EMI. Elsevier Microelectron. Reliab. 2013, 53, 573-581 . [CrossRef]

8. Aiello, O.; Crovetti, P.; Fiori, F. Investigation on the susceptibility of hall-effect current sensors to EMI. In Proceedings of the 10th International Symposium on Electromagnetic Compatibility, York, UK, 26-30 September 2011; pp. 1-4.

9. Satav, S.M.; Agarwal, V. Design and Development of a Low-Cost Digital Magnetic Field Meter With Wide Dynamic Range for EMC Precompliance Measurements and Other Applications. IEEE Trans. Instrum. Meas. 2009, 58, 2837-2846. [CrossRef]

10. Dalessandro, L.; Karrer, N.; Kolar, J.W. High-Performance Planar Isolated Current Sensor for Power Electronics Applications. IEEE Trans. Power Electron. 2007, 22, 1682-1692. [CrossRef]

11. International Standard ISO 11452-4:2005. Road Vehicles-Component Test Method for Electrical Disturbances from Narrowband Radiated Electromagnetic Energy—Part 4: Bulk Current Injection (BCI). Available online: https://www.iso.org/standard/37414.html (accessed on 6 November 2019). 
12. International Standard IS0 11452-3:2016. Road Vehicles-Electrical Disturbances by Narrowband Radiated Electromagnetic Energy, Component Test Method Part 3: Transverse Electromagnetic Mode (TEM) Cell. Available online: https:/ / www.iso.org/standard/66829.html (accessed on 6 November 2019).

13. IEC 623132-4:2006. Integrated Circuits, Measurement of Electromagnetic Immunity_Part 4: Direct RF Power Injection Method. Available online: https://webstore.iec.ch/publication/6510 (accessed on 6 November 2019).

14. Available online: https://www.ansys.com/products/electronics/ansys-maxwell (accessed on 6 November 2019).

15. Fischer Custom Communication (FCC). F-130A-1 Injection Current Probe Characterization: Fischer Custom Communication (FCC); FCC: Torrance, CA, USA, 2004.

16. Fischer Custom Communication (FCC). F-51 Monitor Current Probe Characterization: Fischer Custom Communication (FCC); FCC: Torrance, CA, USA, 2004.

17. Fiori, F.; Musolino, F. Investigation on the effectiveness of the IC susceptibility TEM cell method. IEEE Trans. EMC 2004, 46, 110-114. [CrossRef]

18. Fiori, F.; Musolino, F. Measurement of integrated circuit conducted emissions by using a transverse electromagnetic mode (TEM) cell. IEEE Trans. EMC 2001, 43, 622-628. [CrossRef]

19. Spiegel, R.J.; Joines, W.T.; Blackman, C.F.; Wood, A.W. A method for calculating electric and magnetic fields in TEM cell at ELF. IEEE Trans. Electromagn. Compat. 1987, EMC-29, 265-272. [CrossRef]

20. Redoute, J.M.; Steyaert, M. EMC of Analog ICS; Springer: Berlin, Germany, 2010.

21. Richelli, A.; Matiga, G.; Redoute, J.M. Design of a Folded Cascode Opamp with Increased Immunity to Conducted Electromagnetic Interference in 0.18 um. Elsevier Microelectron. Reliab. 2015, 55, 654-661. [CrossRef]

22. Aiello, O.; Fiori, F. On the Susceptibility of Embedded Thermal Shutdown Circuit to Radio Frequency Interference. IEEE Trans. EMC 2012, 54, 405-412. [CrossRef]

(C) 2019 by the authors. Licensee MDPI, Basel, Switzerland. This article is an open access article distributed under the terms and conditions of the Creative Commons Attribution (CC BY) license (http:/ / creativecommons.org/licenses/by/4.0/). 